\title{
Metastatic Urothelial Carcinoma of the Prepuce and Glans Penis: Suspected Implantation of Non-Muscle-Invasive Bladder Cancer via Urine
}

\author{
Tomoyuki Makino Yasuhide Kitagawa Mikio Namiki \\ Department of Integrative Cancer Therapy and Urology, Kanazawa University Graduate \\ School of Medical Science, Kanazawa, Japan
}

\section{Key Words}

Non-muscle-invasive urothelial carcinoma · Pseudophimosis · Cutaneous implantation

\begin{abstract}
Cutaneous metastatic implantation of non-muscle-invasive urothelial carcinoma via urine is a rare finding, and only few cases have been reported in the literature. Here, we present a case of metastatic urothelial carcinoma of the prepuce and glans penis, which was suspected to be an implantation of non-muscle-invasive bladder cancer via urine. The patient had pseudophimosis of the penis, and contact with urine containing urothelial carcinoma cells was considered to be the cause of the metastatic implantation.

(c) 2014 S. Karger AG, Basel
\end{abstract}

\section{Introduction}

The potential of an implantation of urinary tract urothelial carcinoma via urine has been recognized [1]; however, cutaneous and mucosal implantation, including the prepuce and glans penis, of urothelial carcinoma is clinically extremely rare [2]. Here, we report a case of metastatic urothelial carcinoma of the prepuce and glans penis, which was strongly suspected to be a metastatic implantation of non-muscle-invasive bladder cancer via urine. 
Makino et al.: Metastatic Urothelial Carcinoma of the Prepuce and Glans Penis: Suspected Implantation of Non-Muscle-Invasive Bladder Cancer via Urine

\section{Case Report}

During treatment for hypertension and diabetes mellitus, a 70-year-old male was diagnosed by his general practitioner with renal insufficiency with increased serum creatinine levels of $3.46 \mathrm{mg} / \mathrm{dl}$. Bilateral hydronephrosis and urinary retention were detected on ultrasonography, and the patient was referred to our department. Computed tomography (CT) and magnetic resonance imaging (MRI) revealed widespread papillary tumors on the bladder wall and urine cytology had positive findings. Transurethral resection of the bladder tumor (TUR-BT) was performed, and the pathological findings revealed urothelial carcinoma of the bladder (pTa, low grade). The patient's bilateral hydronephrosis and renal function were improved by the insertion of a urethral catheter; hydronephrosis and renal insufficiency did not occur after the removal of the urethral catheter. After TUR-BT, 4 instances of tumor recurrence were observed, and additional TUR-BTs were performed during a 2.5-year observation period. Each pathological finding revealed low-grade, non-muscle-invasive bladder carcinoma.

Six months after the last TUR-BT, when the patient was 73 years old, cystoscopic examination showed multiple papillary pedunculated tumors. TUR-BT was not performed immediately because of comorbidities, and urine cytology continuously revealed positive findings for urothelial carcinoma cells. TUR-BT was again performed after 8 months of cystoscopic examination. The patient had pseudophimosis of the penis, and the examination of the prepuce and glans penis during TUR-BT revealed several papillary tumors with a maximum size equivalent to that of the tip of the small finger. TUR-BT, circumcision, and electrocoagulation of the tumor on the glans penis were performed. Pathological findings revealed low-grade, non-muscle-invasive urothelial carcinoma (fig. 1) and metastatic urothelial carcinoma of the prepuce (fig. 2, fig. 3). After surgery, no recurrence was detected during a 2-year observation period.

\section{Discussion}

A previous review of the literature for reports of cutaneous metastases from genitourinary malignancies defined 4 basic mechanisms of metastatic dissemination by which the skin could be accessed: (a) direct invasion from the underlying neoplasm; (b) implantation from an operative scar; (c) spread through the lymphatics, and/or (d) hematogenous spread [1]. Although the review reported that these findings occurred in advanced disease in almost all cases, implantation from an operative scar may indicate the potential for a tumor implantation of urothelial carcinoma via urine. In addition, there were several reports of seeding of urothelial carcinoma outside the urinary tract after iatrogenic surgical procedures [3].

On the other hand, a metastatic cutaneous implantation of non-muscle-invasive urothelial carcinoma via urine not caused by iatrogenic procedures is clinically extremely rare; there have been only few reports $[2,4,5]$. In our case, the pathological findings of papillary tumors from the prepuce resembled those of the tumors from the bladder. CT revealed no distant metastases, and a metastatic implantation of the prepuce and glans penis was strongly suspected. Furthermore, the pseudophimosis observed in our patient has only been reported in 1 patient so far [2]. In some previous cases, contact with urine containing urothelial carcinoma cells was considered to be the cause of a metastatic implantation of the labia minor [4,5]; a similar mechanism may be involved in pseudophimosis. Skin care is 
Makino et al.: Metastatic Urothelial Carcinoma of the Prepuce and Glans Penis:

Suspected Implantation of Non-Muscle-Invasive Bladder Cancer via Urine

important in patients with urothelial carcinoma whose urine may continuously contain malignant cells.

\section{Disclosure Statement}

The authors have no conflicts of interest to declare.

\section{References}

1 Mueller TJ, Wu H, Greenberg RE, Hudes G, Topham N, Lessin SR, Uzzo RG: Cutaneous metastases from genitourinary malignancies. Urology 2004;63:1021-1026.

-2 Miyamoto T, Ikehara A, Araki M, Akaeda T, Mihara M: Cutaneous metastatic carcinoma of the penis: suspected metastasis implantation from a bladder tumor. J Urol 2000;163:1519.

-3 Andersen JR, Steven K: Implantation metastasis after laparoscopic biopsy of bladder cancer. J Urol $1995 ; 153: 1047$.

4 Yu DS, Harn HJ, Lee WH, Ma CP: Transitional cell carcinoma of the labia minor: evidence of contact implantation of bladder cancer. J Urol 1996;155:1699.

5 Ogiso S, Maeno A, Yamashita M, Souma T, Nakamura K, Okuno H: Micturitional disturbance due to labial adhesion as a cause of bladder urothelial carcinoma. Int J Urol 2006;13:1454-1455.

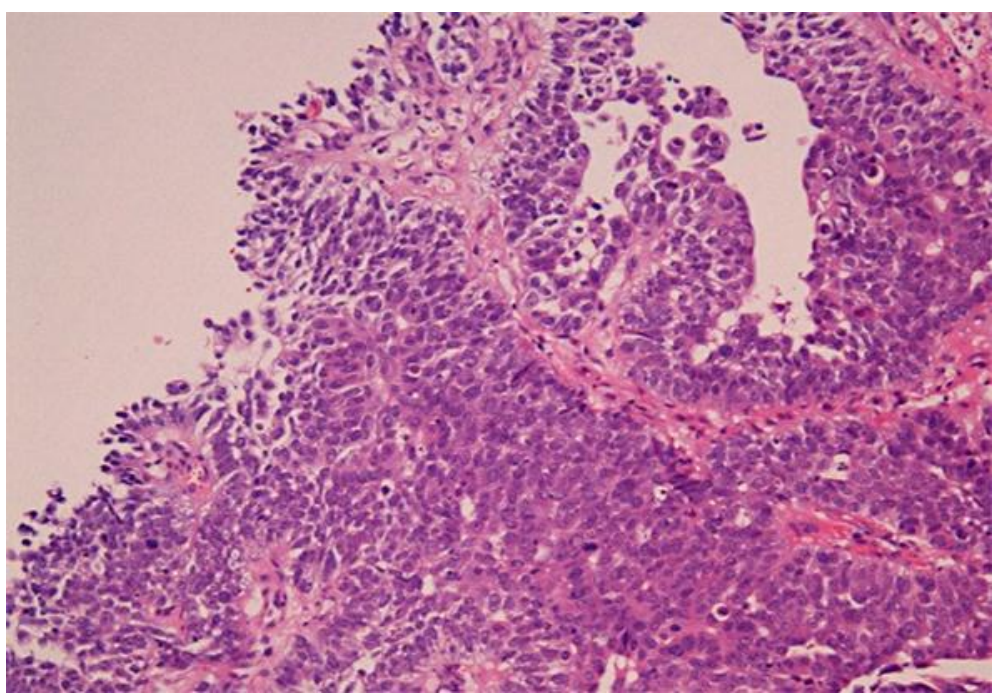

Fig. 1. Microscopic appearance of the TUR-BT specimen of the papillary bladder tumor after HE staining. 


\section{Case Reports in Oncology}

\begin{tabular}{l|l}
\hline Case Rep Oncol 2014;7:509-512 \\
\hline DOI: 10.1159/000365749 & $\begin{array}{l}\text { @ 2014 S. Karger AG, Basel } \\
\text { www.karger.com/cro }\end{array}$ \\
\hline
\end{tabular}

Makino et al.: Metastatic Urothelial Carcinoma of the Prepuce and Glans Penis: Suspected Implantation of Non-Muscle-Invasive Bladder Cancer via Urine

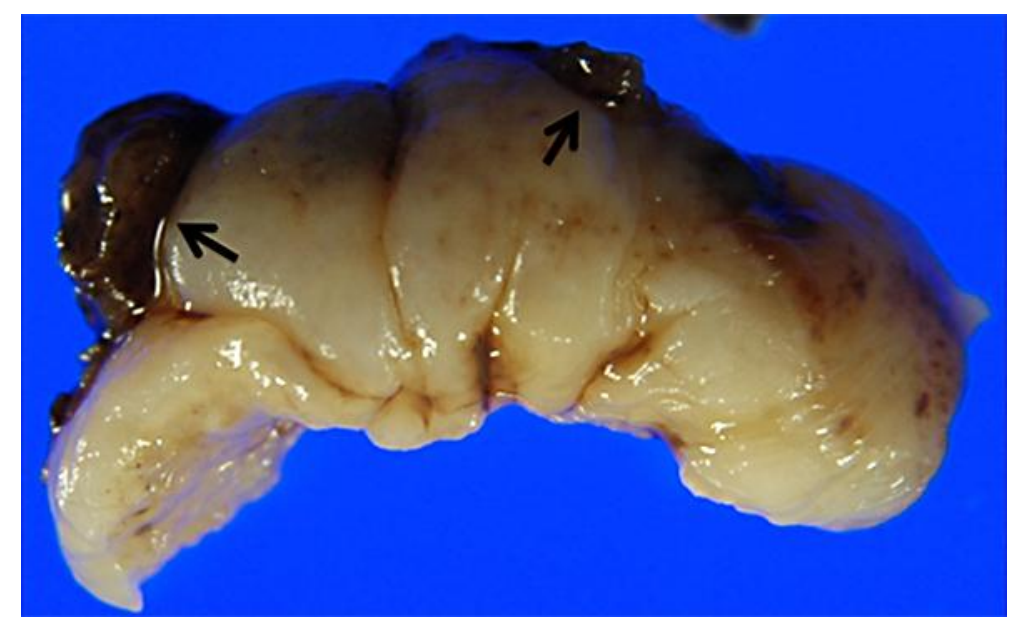

Fig. 2. Macroscopic appearance of the prepuce after circumcision. Arrows indicate the tumors on the prepuce.

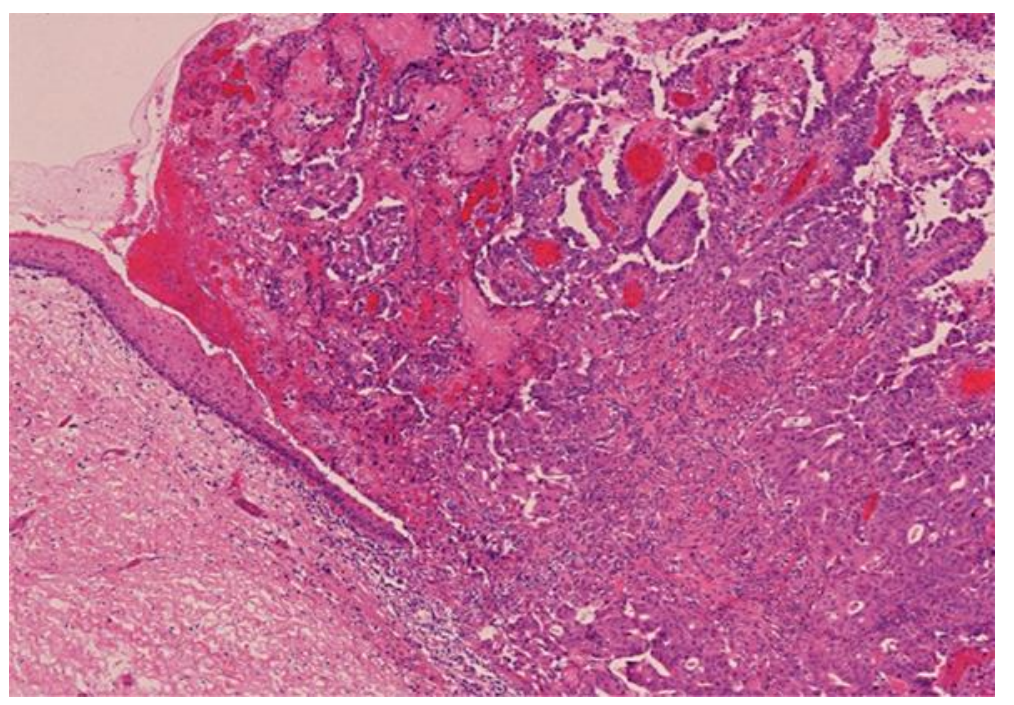

Fig. 3. Microscopic appearance of the tumor on the prepuce showing the papillary growth of urothelial carcinoma. 